Original Article

\section{Assessment of different enzymatic virulence traits contributing to pathogenicity of Candida albicans.}

\author{
Sadia Nazeer', Sehar Afshan Naz $^{2}$, Arif Zubair ${ }^{3}$, Maryam \\ Shafique $^{2}$, Shaheen Sharafat ${ }^{4}$, Samina Baig ${ }^{4} \&$ Gul Jabeen ${ }^{2}$ \\ ${ }^{1}$ Department of Biomedical Engineering, Sir Syed University of Engineering and \\ Technology, Karachi- Pakistan. \\ ${ }^{2}$ Department of Microbiology, Federal Urdu University of Arts, Science and \\ Technology, Karachi-Pakistan. \\ ${ }^{3}$ Department of Environmental Sciences, Federal Urdu University of Arts, Science and \\ Technology, Karachi-Pakistan. \\ ${ }^{4}$ Dow University of Health Sciences, Karachi-Pakistan.
}

Doi: 10.29052/JJEHSR.v8.i3.2020.129-138

\section{Corresponding Author Email:}

seharafshan@fuuast.edu.pk

Received 02/05/2020

Accepted 26/08/2020

First Published 01/09/2020

\section{(c) (1)}

(C) The Author(s). 2020 Open Access This article is distributed under the terms of the Creative Commons Attribution 4.0 International License (http://creativecommons.org/licenses/by/4.0/)

OPEN

ACCESS

\begin{abstract}
Background: Candidiasis is regarded as one of the frequently occurring fungal infections of humans elicited by yeast that belongs to the genus Candida. Among the different species of Candida, Candida albicans is the most pathogenic specie. The pathogenicity of this specie depends on various virulence factors. This study was designed to determine the activity of some important enzymatic virulence factors such as phospholipase, proteinase and hemolysin produced by Candida albicans strains.

Methodology: A total of 300 clinical isolates of C. albicans were obtained and identified by conventional standard diagnostic criteria. For the evaluation of phospholipase production, the egg yolk agar medium was employed. The minimum agar medium was used for proteinase activity and for hemolysin production, blood plate assay was used. The results were statistically analyzed by SPSS 16.0 version software.

Results: Of a total of 300 isolates reviewed, the majority of the isolates were recovered from females, i.e. 234 (78\%); however, the number of isolates obtained from males were $66(22 \%)$. Amongst all the strains of C. albicans, phospholipase and hemolysin enzymes were produced primarily by $89.3 \%$ of the total C. albicans strains. While proteinase enzyme was produced by $86 \%$ of the total C. albicans isolates. A p-value $<0.05$ was measured as statistically significant.

Conclusion: From the existing study, it can be determined that these enzymes are produced by most of the pathogenic strains of $C$. albicans. The detection of these virulence enzymes is tremendously significant in designing of drugs to combat these opportunistic infections in future.
\end{abstract}

\title{
Keywords
}

Candida Albicans, Virulence Factors, Phospholipase, Proteinase, Hemolysin.

\section{Check for updates}




\section{Introduction}

Candidiasis is a type of fungal infection caused by yeast, which belongs to the genus Candida. Although different Candida species are present, but Candida albicans is the most infective species which is responsible for more than half of the cases of candidiasis in various countries ${ }^{1,2}$. C. albicans is an expedient organism that has established a diverse range of virulence traits that convert this fungus from an innocuous commensal into an infectious microorganism. These virulence factors involve the manifestation of invasins and adhesins on the surface of the cell, phenotypic switching, biofilm formation, morphological change among yeast and hyphal structures and the production of extracellular hydrolytic enzymes ${ }^{3,4}$. The most dominating virulence feature of $C$. albicans in the release of extracellular hydrolytic enzymes as they assist in assimilating molecules for nutrition supply. Furthermore, these enzymes can also help in tissue invasion by the degradation of the host cell membrane. Different extracellular enzymes that are associated with the virulence of $C$. albicans are proteinases, chondroitinases, hemolysins, lipases and hyaluronidases ${ }^{5}$.

The most considerable extracellular hydrolytic enzymes originated from C. albicans are phospholipases as they perform an essential function in the overgrowth of C. albicans, and these enzymes help in the attachment, tissue invasion and penetration of the host. Phospholipases possibly intricate in the process of disruption of the cell membrane that happens during the course of the invasion of the host cell then causes the adherence and penetration into the cell. The host cell membrane is damaged by phospholipases through splitting phospholipids, thus resulting in cell lysis 6 .

The Secreted Aspartyl Proteinases (SAPs) released from $C$. albicans can hydrolyze different types of human proteins on the injury site like hemoglobin, albumin, keratin, collagen, secretory immunoglobulin A $(\lg A-s)$, endothelial cell proteins, extracellular matrix proteins, mucin, different molecules in the immune system, various clotting and coagulation elements. This proteolytic activity has been linked to tissue incursion, and thus it causes tissue penetration by Candida species $^{7,8}$.

Hemolysin causes the lysis of erythrocytes that ensues the liberation of iron, which is a significant growth factor for fungi mostly throughout the fungal infections. In the cases of systemic candidiasis, the production of hemolysin causes the acquirement of iron that assists in the incursion of hyphae as in the hyphae of C. albicans, a larger amount of receptors of hemoglobin are present as compared to the yeast form of Candida. As, in C. albicans, during disseminated candidiasis, the production of hemolysin is connected with the iron uptake that helps in the incursion of hyphae. $\ln C$. albicans, the release of hemolysin causes the iron uptake, which induces phase switching ${ }^{9}$.

Because of the expanding prevalence of intrusive infections caused by C. albicans, attention towards their virulence factors has amplified. Accordingly, looking towards the importance of these enzymatic virulence features, the contemporary research aimed to determine the production of some enzymatic virulence features such as phospholipase, hemolysin and proteinase from endogenous isolates of $C$. albicans obtained from different clinical species.

\section{Methodology}

The present research work was conducted at the Department of Microbiology, Federal Urdu University of Arts, Science and Technology, Karachi, with the collaboration of Dow University of Health Sciences (DUHS), Karachi. A total of 300 clinical Candida albicans isolates were obtained from DUHS during one year period. These $C$. albicans isolates were reidentified by using standard diagnostic criteria ${ }^{10}$. The identification of the $C$. albicans isolates was done by performing various conventional tests like gram staining, wet mount technique and germ tube test. The biochemical identification was done by carbohydrates fermentation and assimilation tests. Moreover, the isolates were also grown on Cornmeal Tween80 media and Chromogenic media. However, for the differentiation of $C$. 
albicans and C. dubliniensis, the growth of isolates was also assessed by growing them at $45^{\circ} \mathrm{C}$. Moreover, the doubtful isolates were further identified by using Remel RapID TM yeast plus kit. The results were matched with a control culture of C. albicans ATCC 14053.

In the current research work, 300 clinical isolates of C. albicans were screened for the secretion of enzymatic virulence factors. The activity of phospholipase from $C$. albicans isolates was examined by egg yolk agar assay ${ }^{11}$. For the detection of phospholipase activity, 24 hrs fresh Sabouraud's dextrose agar (SDA) grown $C$. albicans culture was stabbed into egg yolk agar medium and was incubated at $37^{\circ} \mathrm{C}$ for four days. The proteolytic analysis was carried out in minimal medium $\operatorname{agar}^{12}$. For the detection of proteinase activity, 24 hrs of fresh SDA grown C. albicans culture was stabbed on the test medium then it was incubated at $37^{\circ} \mathrm{C}$ for four days. Hemolysin production was assessed with the blood plate assay $^{13}$. For the detection of hemolysin production, 24 hrs of fresh SDA grown C. albicans culture was stabbed on the test medium then the culture was incubated at $37^{\circ} \mathrm{C}$ for $48 \mathrm{hrs}$.

The enzymatic activity was assessed according to the Pz index. The value of Pz was calculated by the ratio of colony diameter to the diameter of the colony plus zone of precipitation (in $\mathrm{mm}$ ), and it was measured manually for each isolate. The zone of enzymatic activity was calculated in accordance with Price method ${ }^{14}$.

\section{Zone of enzymatic activity $(\mathbf{P z})=$}

Diameter of colony

\section{Diameter of colony + zone of precipitation}

Enzymatic activity values were scored and categorized as follows:

- $\quad \mathrm{Pz}=1$ : no enzymatic activity (-ve)

- $\quad P z=0.9-0.99$ : weak activity $(+)$

- $\mathrm{Pz}=0.8-0.89$ : mild activity $(++)$

- $\quad \mathrm{Pz}=0.7-0.79$ : strong activity $(+++)$

- $\mathrm{Pz}=<0.69$ : very strong activity $(++++)$

The assays were accomplished in triplicates, and enzymatic activity scores were noted as the average of the three assessments. The SPSS software version 16 was used to analyze the facts and figures. Chi-square test, co-relation coefficient and ANOVA were employed in the present study. A $p$-value of $<0.05$ was measured statistically noteworthy.

\section{Result}

Of a total of 300 isolates reviewed in this study, the majority of the isolates were recovered from females i.e. 234 (78\%) as compared to males 66 (22\%). Most of the C. albicans isolates were obtained from urine samples ( $57 \%$ ) ensued by high vaginal swab (22\%). The rate of isolation of $C$. albicans from the urine sample of women was remarkably higher (75\%) by way of comparison to males was $25 \%$ (Table 1). The dissimilarities in the prevalence of isolates of $C$. albicans among men and women from various clinical specimens were statistically significant at $p=0.001$. 


\section{Table 1: Distribution of isolated Candida albicans strains in accordance with the clinical samples.}

\begin{tabular}{lcccccc}
\hline Clinical Samples & \multicolumn{2}{c}{$\begin{array}{c}\text { Clinical specimens } \\
\text { collected from male } \\
\text { patients }\end{array}$} & $\begin{array}{c}\text { Clinical specimens } \\
\text { collected from female } \\
\text { patients }\end{array}$ & \multicolumn{2}{c}{$\begin{array}{c}\text { Total number of } \\
\text { clinical isolates }\end{array}$} \\
\cline { 2 - 8 } & No. & $* \%$ & No. & $* \%$ & No. & $\%$ \\
\hline Urine & 42 & 24.5 & 129 & 75.4 & 171 & 57 \\
\hline HVS & 0 & 0 & 66 & 100 & 66 & 22 \\
\hline Sputum & 14 & 38 & 23 & 62.1 & 37 & 12.3 \\
\hline Blood & 4 & 57.1 & 3 & 43 & 7 & 2.3 \\
\hline $\begin{array}{l}\text { Cerebral Spinal } \\
\text { Fluid }\end{array}$ & 0 & 0 & 1 & 100 & 1 & 0.3 \\
\hline Tracheal Fluid & 1 & 16.6 & 5 & 83.3 & 6 & 2 \\
\hline $\begin{array}{l}\text { Oral And Ear } \\
\text { Swabs }\end{array}$ & 3 & 50 & 3 & 50 & 6 & 2 \\
\hline Pus & 1 & 20 & 4 & 80 & 5 & 1.8 \\
\hline $\begin{array}{l}\text { Central Venous } \\
\text { Pressure Tip }\end{array}$ & 1 & 100 & 0 & 0 & 1 & 0.3 \\
\hline Total & 66 & 22 & 234 & 78 & 300 & 100 \\
\hline
\end{tabular}

*HVS-High Vaginal Swab; CSF-Cerebrospinal Fluid.

*\% calculated from total number of clinical isolates obtained from particular specimen.

*Statistical analysis by Chi-Square Test revealed a p-value $0.001<0.05$.

The changes in the prevalence of $C$. albicans isolates among men and women in different age groups were statistically significant $(p=0.045<0.05)$.

\section{$\%$ of $\mathrm{C}$. albicans isolates from females $\%$ of C. albicans isolates from males}

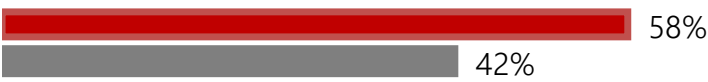

$60-79$

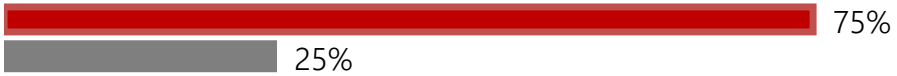

40-59

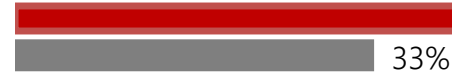

$67 \%$

20-39

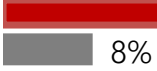

Figure 1: Distribution of Candida albicans isolates in different age groups. 
The highest rate of isolation of $C$. albicans was found in the patient's age group ranged from 20 to 39 years in females and age group ranged from 80 to 99 years in males. Interestingly, the lowest isolation rate was observed among females age group ranged from 80 to 99 years and in males age group ranged from 20-39 years (Figure 1).

Among all the isolates of C. albicans, phospholipase and hemolysin enzymes were produced by $89.3 \%$ (268/300) of the total C. albicans strains. While proteinase enzyme was produced by $86 \%$ (258/300) of the total C. albicans isolates (Table 2).

Table 2: Enzymatic virulence activities of isolated Candida albicans according to the site of origin.

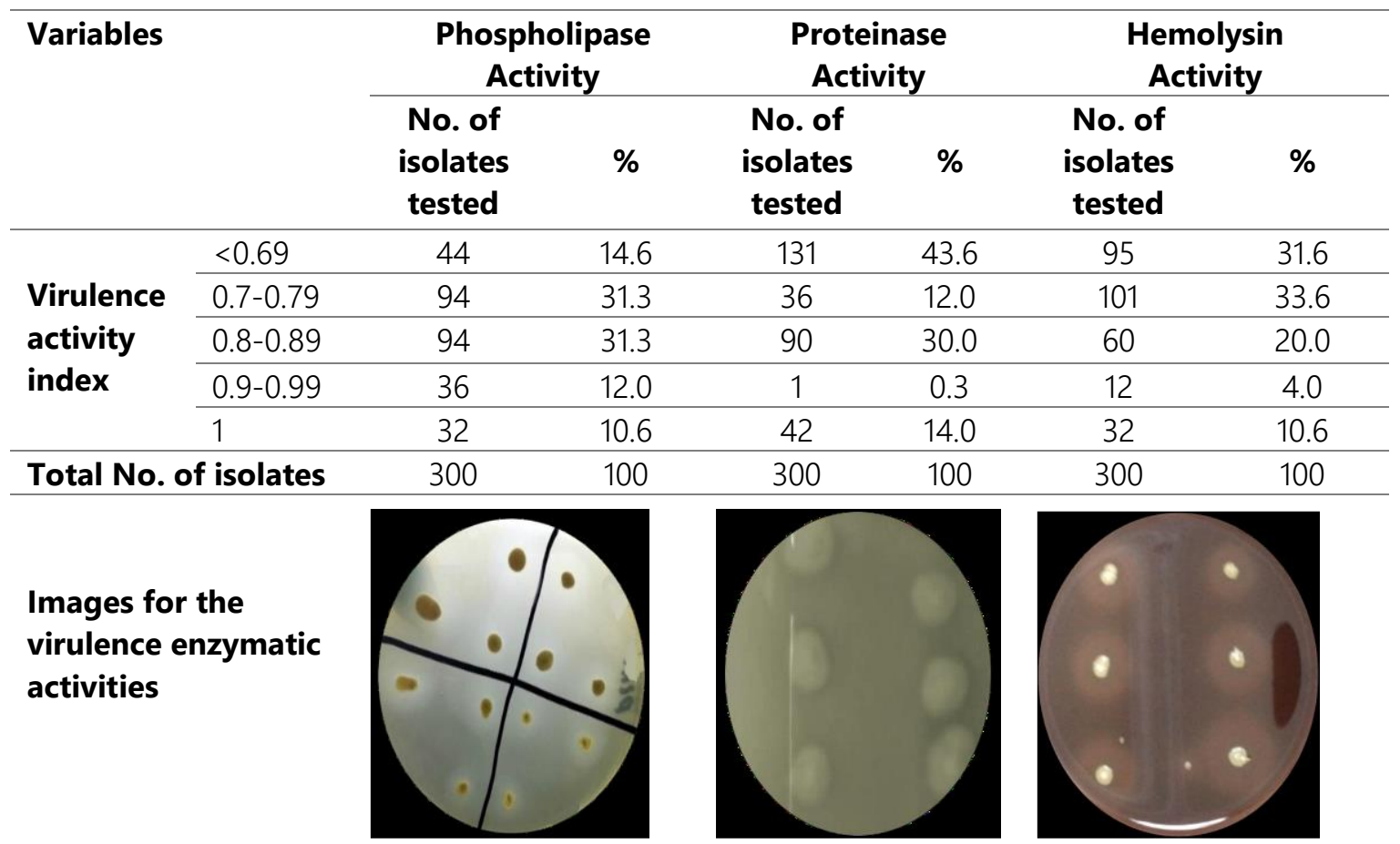

${ }^{*} n=$ number of isolates.

$* \%(n)=$ number of isolates producing enzymatic activity from each specimen with total number of isolates from each specimen.

For phospholipase, the zone of precipitation was perceived encircling the colony on the plate. In the case of proteinase enzyme, the deterioration of protein was observed as cloudiness of the agar, which can be observed as a region of proteolysis encircling the colony. While for hemolysin, a zone of hemolysis was observed around the colonies of $C$. albicans (Table 3). 
Table 3: Distribution of $\mathrm{Pz}$ value of enzymatic virulence factors among the isolates of $C$. albicans.

\begin{tabular}{lcccccc}
\hline $\begin{array}{l}\text { Clinical specimens } \\
\text { (n) }\end{array}$ & $\begin{array}{c}\text { Phospholipase Activity } \\
\text { No. of } \\
\text { isolates (n) }\end{array}$ & $\begin{array}{c}\text { P } \mathbf{( n )} \\
\text { Proteinase Activity }\end{array}$ & $\begin{array}{c}\text { No. of } \\
\text { isolates (n) }\end{array}$ & $\begin{array}{c}\text { Hemolysin Activity } \\
\text { (n) }\end{array}$ & $\begin{array}{c}\text { No. of } \\
\text { isolates (n) }\end{array}$ & \% (n) \\
\hline Urine (171) & 163 & 95.3 & 152 & 88.8 & 155 & 90.6 \\
\hline HVS (66) & 56 & 84.8 & 53 & 80.3 & 58 & 87.8 \\
\hline Sputum (37) & 28 & 75.6 & 29 & 78.3 & 32 & 86.4 \\
\hline Blood (7) & 7 & 100.0 & 6 & 85.7 & 7 & 100.0 \\
\hline CSF (1) & 1 & 100.0 & 1 & 100.0 & 1 & 100.0 \\
\hline Tracheal Fluid (6) & 4 & 66.6 & 5 & 83.3 & 3 & 50.0 \\
\hline $\begin{array}{l}\text { Oral \& Ear Swabs } \\
\text { (6) }\end{array}$ & 4 & 66.6 & 6 & 100.0 & 6 & 100.0 \\
\hline Pus (5) & 4 & 80.0 & 5 & 100.0 & 5 & 100.0 \\
\hline $\begin{array}{l}\text { Central Venous } \\
\text { Pressure Tip (1) }\end{array}$ & 1 & 100.0 & 1 & 100.0 & 1 & 0.0 \\
\hline Total=300 & 268 & 89.3 & 258 & 86.0 & 268 & 89.3 \\
\hline
\end{tabular}

*HVS-High Vaginal Swab; CSF-Cerebrospinal Fluid.

The maximum virulence enzymatic production was found in the C. albicans isolates obtained from urine samples followed by high vaginal swab, sputum, blood and other clinical specimens (Table 2). A considerable connection was perceived between the clinical specimen and phospholipase activity of $C$. albicans isolates $(p=0.006<0.05)$. But there is no significant relationship between the clinical specimen and proteinase activity of $C$. albicans isolates ( $p=0.637>0.05$ ). No considerable linkage was found between the clinical specimen and hemolysin activity of $C$. albicans isolates ( $p=0.604>0.05$ ). Among 300 isolates of C. albicans, 230 (76.6\%) C. albicans isolates were positive for all the three enzymatic activities (Figure 2). While 230 (76.6\%) isolates demonstrated positivity for both phospholipase and proteinase activities. Though $79.6 \%$, i.e. 239 isolates of $C$. albicans, presented the enzymatic measures for both phospholipase and hemolysin. In 243 (81\%) of the isolates of $C$. albicans, the enzymatic activities of proteinase and hemolysin were observed. Lastly, no consequential alliance was found between phospholipase, hemolysin and proteinase activities of isolates of $C$. albicans. However, a strong correlation is found between phospholipase and proteinase activities only $(p=0.05 \leq 0.05)$. 


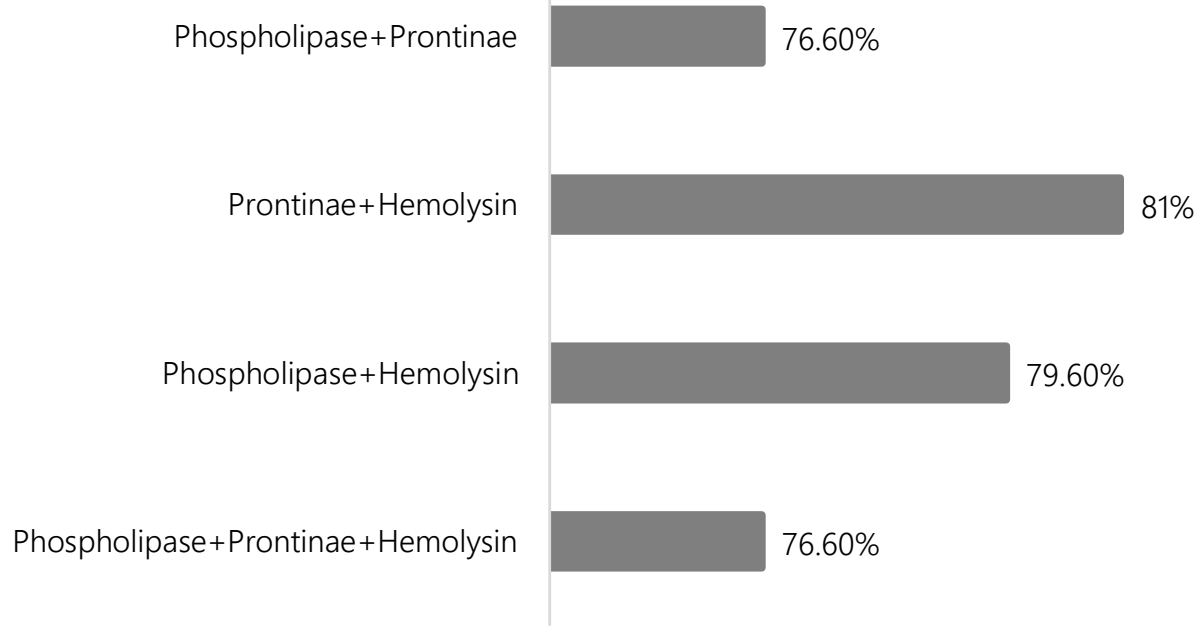

Figure 2: Virulence factors associations among the isolates of Candida albicans.

\section{Discussion}

Candida albicans is one of the most substantial and expedient fungal pathogens associated with an increased existence in immunosuppressed patients. The increasing morbidity and mortality associated with candidiasis are because of lower efficacy, higher toxicity and emergence of resistance against currently available antifungal drugs $^{15-17}$. In order to develop new alternatives for conventional antifungal drugs, the virulence factors and their inhibitors have been extensively explored and characterized in the last few years ${ }^{18}$. Among these approaches, certain anti-secreted hydrolytic enzymes, such as inhibitors of fungal proteases, are considered a significant approach to the safe treatment of Candidiasis ${ }^{19-21}$.

C. albicans pathogenicity is intervened by numerous virulence factors, the most significant of which is the secretion of hydrolytic enzymes (e.g. phospholipases, proteases and hemolysins) that promote colonization and tissue incursion ${ }^{22}$. The current study was designed to evaluate the in vitro production of some virulence enzymes such as phospholipase, hemolysin and proteinase in clinical isolates of $C$. albicans.
The present study revealed the maximum isolation of $C$. albicans strains from female patients. Most of the strains were isolated from urine (57\%) and high vaginal swab (22\%) (Table 1). As C. albicans is a normal flora in the vagina, but in some cases, it may also cause vaginitis. Different factors that contribute to candidal growth in females are hormonal contraceptives, antibiotic cure, pregnancy, diabetes, debilitated immune system, and HIV/AIDS ${ }^{23}$. In the study, most of the isolates of $C$. albicans were obtained from younger females of age group (20-39) (Figure 1), which was in accordance with a previous study ${ }^{24}$. It has been understood that the increasing number of $C$. albicans amongst the young age group women may be because of improved functional variations in estrogen and the abundant glycogen volume of the vaginal mucosal membrane providing adequate provision of functional sugar that contribute to the spread of $C$. albicans especially in pregnant women ${ }^{24}$.

Numerous features have been combined to the greater tendency of colonization of $C$. albicans in females as compared to their male correspondents but in the current study, male patients showed the highest prevalence of $C$. albicans isolates in the age group (80-99) (Figure 1). This high prevalence 
might be due to the weak immune responses during old ages. Additionally, other features like diminished hepatic and renal functions also enrich candida colonization in this age group ${ }^{25}$.

In the current study, the phospholipase activity (Pz) was observed in 268 (89.3\%) isolates of the total isolates, while 138 (46\%) of these strains showed a higher $\mathrm{Pz}$ value of $\leq 0.79 \mathrm{~mm}$ (Table 3 ). These findings simulate with preceding studies where phospholipase activity was stated in $75 \%$ to $100 \%$ isolates of $C$. albicans isolated from varied origin sites and from several types of patients ${ }^{8}$. According to research conducted in $2014,87.7 \%$ of the isolates showed phospholipase activity 26 . The parallel percentage (77\%) of phospholipase activity was also revealed in another study, which suggests that the isolates used in the present study were comparatively more hazardous than the prior researches ${ }^{24}$. In the current research, hemolysin activity was seen in $89.3 \%$ isolates; however, the maximum hemolytic activity ( $\mathrm{Hz} \leq 0.79 \mathrm{~mm}$ ) was observed in $65.3 \%$ of the isolates (Table 3 ). The enzymatic measure of hemolysin in the $C$. albicans strains has been well documented in the studies previously conducted ${ }^{26}$.

Proteinase production, another virulence trait, was also determined by $86 \%$ of the isolates of $C$. albicans, whereas $55.6 \%$ of the strains showed a higher proteinase value of $\leq 0.79 \mathrm{~mm}$ (Table 3 ). These findings are in the support of a research conducted in 2017, where $81.1 \%$ of the isolates of $C$. albicans presented the activity of proteinase ${ }^{27}$. Similarly, according to a study, $79.5 \%$ of $C$. albicans strains showed positivity for proteinase enzyme ${ }^{28}$. The concurrent production of phospholipase, proteinase and hemolysin in the same strains have been examined by rare contemplates. In the extant study, $76.6 \%$ of the total isolates produced phospholipase, proteinase and hemolysin simultaneously (Figure 2) which is in accordance with the outcomes obtained from a previous study $^{29}$. In an alternative analogous study, estimates of the enzymatic activity suggested that $100 \%$ of the isolates of C. albicans were virulent enzyme producers ${ }^{9}$. However, the existent analysis suggests that $2 \%$ of the total C. albicans isolates exhibited no enzymatic output of proteinase, phospholipase and hemolysin.

Studies from the numerous geographical regions demonstrate that different levels of phospholipase activity can be perceived from different counties. According to research, conducted in 2003, investigators revealed that virulent influences could be related to infection type and geographic area $^{30}$. Contradictory, as stated by a study, $53.08 \%$ verified C. albicans showed phospholipase activity that instigated from healthy adults ${ }^{31}$. In the present study, 163/171 (95.3\%) isolates obtained from urine displayed phospholipase production, which is followed by $56 / 66$ (85\%) isolates obtained from high vaginal swabs. Similarly, 152/171 (88.8\%) isolates procured from urine showed activity of proteinase enzyme. Likewise, 155/171 (90.6\%) isolates acquired from urine exhibited production of hemolysin also (Table 2). Probably the inconstant levels of enzymatic activities in our study are associated with the different clinical sources of $C$. albicans.

\section{Conclusion}

The results of the present research revealed the significance of enzymatic virulence factors in the pathogenicity of $C$. albicans. This investigation also depicted the association of infection site with the production of virulence factors by these fungi. These virulence factors also indicate the invasiveness of the infection. Therefore, the timely revelation of virulence enzymes production by $C$. albicans is valuable in making an appropriate clinical verdict.

\section{Conflicts of Interest}

None.

\section{Acknowledgement}

We are thankful to Dr. Mushtaq Hussain, Assistant Professor, Dow University of Health Sciences, Karachi and Dr. Nusrat Jabeen, Associate Professor, Department of Microbiology, University of Karachi, Karachi for their sincere support throughout this study. 


\section{Funding}

This study was partially funded by Higher Education Commission Pakistan through NRPU: 4497, awarded to corresponding author.

\section{References}

1. Singh DK, Toth $R$, Gacser A. Mechanisms of pathogenic Candida species to evade the host complement attack. Front Cell Infect Microbiol. 2020;10:1-9.

2. Caggiano G, Lovero G, De Giglio O, Barbuti G, Montagna O, Laforgia N, Montagna MT. Candidemia in the neonatal intensive care unit: a retrospective, observational survey and analysis of literature data. Bio Med Res Int. 2017; 2017: 1-12.

3. Galocha M, Pais P, Cavalheiro M, Pereira D, Viana R, Teixeira MC. Divergent approaches to virulence in $C$. albicans and C. glabrata: Two sides of the same coin. Int J Mol Sci. 2019; $20: 2345$.

4. Staniszewska M. Virulence factors in Candida species. Curr Protein Pept Sc. 2020; 21:313-23.

5. Pereira CA, Costa AC, Silva MP, Back-Brito GN, Jorge AO. Candida albicans and virulence factors that increases its pathogenicity. The Battle against Microbial Pathogens: Basic Science, Technological Advances and Educational Programs (A. MéndezVilas, Ed.); Microbiology Series. 2015:631-636.

6. Deepa K, Jeevitha T, Michael A. In vitro evaluation of virulence factors of Candida species isolated from oral cavity. J Microbiol Antimicrob. 2015; 7:28-32.

7. Kalaiselvi G. Role of phospholipase and proteinase as virulence factors of Candida albicans isolated from clinical samples of inpatients in a tertiary care hospital. Int J Scientific Res Rev. 2014; 3: 167 - 76.

8. Lima JS, Braga KR, Vieira CA, Souza WW, ChávezPavoni JH, Araújo CD, Goulart LS. Genotypic analysis of secreted aspartyl proteinases in vaginal Candida albicans isolates. Jornal Brasileiro de Patologia e Medicina Laboratorial. 2018;54:28-33.

9. Rossoni RD, Barbosa JO, Vilela SF, Jorge AO, Junqueira JC. Comparison of the hemolytic activity between C. albicans and non-albicans Candida species. Braz Oral Res. 2013; 27:484-489.

10. Deorukhkar SC, Saini S. Laboratory approach for diagnosis of candidiasis through ages. Int J Curr Microbiol Appl Sci. 2014; 3:206-218.

11. Ellepola AN, Samaranayake LP, Khan ZU. Extracellular phospholipase production of oral Candida albicans isolates from smokers, diabetics, asthmatics, denture wearers and healthy individuals following brief exposure to polyene, echinocandin and azole antimycotics. Braz J Microbiol. 2016; 47:911-916.

12. Ishida K, Ueda-Yamaguchi M, Yamada-Ogatta SF, Ueda-Nakamura T, Svidizinsk TI, Nakamura CV. Characterization of Candida spp. isolated from vaginal fluid: identification, antifungal susceptibility, and virulence profile. Acta Sci Health Sci. 2013; 35:18.

13. Noori $M$, Dakhili $M$, Sepahvand $A$, Davari $N$. Evaluation of esterase and hemolysin activities of different Candida species isolated from vulvovaginitis cases in Lorestan Province, Iran. Curr. Med. Mycol. 2017; 3:1-5.

14. Price MF, Wilkinson ID, Gentry LO. Plate method for detection of phospholipase activity in Candida albicans. Sabouraudia: J Med Vet Mycol. 1982; 20:714.

15. Pierce CG, Lopez-Ribot JL. Candidiasis drug discovery and development: new approaches targeting virulence for discovering and identifying new drugs. Exp Opin Drug Disc.2013; 8: 1117-1126.

16. Clatworthy $A E$, Pierson $E$, Hung DT. Targeting virulence: a new paradigm for antimicrobial therapy. Nat Chem Biol. 2007; 3: 541-548.

17. Cowen LE. The evolution of fungal drug resistance: modulating the trajectory from genotype to phenotype. Nat Rev Microbiol. 2008; 6:187-198.

18. Gauwerky K, Borelli C, Korting HC. Targeting virulence: a new paradigm for antifungals. Drug Disc Today.2009;14: 214-222.

19. Hoeg L, Thoma-Greber E, Rocken M, Korting HC. HIV protease inhibitors influence the prevalence of oral candidosis in HIV-infected patients: a 2-year study. Mycoses. 1998; 41: 321-325.

20. Cauda R, Tacconelli E, Tumbarello $M$ et al. Role of protease inhibitors in preventing recurrent oral candidosis in patients with HIV infection: a prospective case-control study. J Acquir Immune Defic Syndr. 1999; 21: 20-25.

21. Ahmad Khan MS, Alshehrei F, Al-Ghamdi SB, Bamaga MA, Al-Thubiani AS, Alam MZ. Virulence and biofilms as promising targets in developing antipathogenic drugs against candidiasis. Future Sci OA. 2020 ; 6 :FSO440. Published 2020 Feb 3. doi:10.2144/fsoa-2019-0027.

22. de Paula Menezes R, de Melo Riceto ÉB, Borges AS, de Brito Röder DV, dos Santos Pedroso R.Evaluation of virulence factors of Candida albicans isolated from HIV-positive individuals using HAART. Arch Oral Biol. 2016; 66:61-65.

23. Jeanmonod $R$, Jeanmonod $D$, Christopherson $N$, Spivey R. Candidiasis, Vaginal (Vulvovaginal Candidiasis). Stat Pearls. 2018. 
24. Altayyar IA, Alsanosi AS, Osman NA. Prevalence of vaginal candidiasis among pregnant women attending different gynecological clinic at South Libya. Eur J Exp Biol. 2016; 6:25-29.

25. Flevari A, Theodorakopoulou $M$, Velegraki $A$, Armaganidis A, Dimopoulos $G$. Treatment of invasive candidiasis in the elderly: a review. Clin Interv Aging. 2013; 8:1199-1208.

26. Deorukhkar SC, Saini S, Mathew S. Non-albicans Candida infection: an emerging threat. Interdiscip Perspect Infect Dis. 2014:1-7.

27. Lahkar V, Saikia L, Patgiri SJ, Nath R, Das PP. Estimation of biofilm, proteinase \& phospholipase production of the Candida species isolated from the oropharyngeal samples in HIV-infected patients. Indian J Med Res. 2017; 145:635-640.

28. Jasim ST, Flayyih MT, Hassan AA. Isolation and identification of Candida spp. from different clinical specimens and study the virulence factors. World J Pharmaceut Sci. 2016; 5:121-137.

29. Sachin CD, Ruchi K, Santosh S. In vitro evaluation of proteinase, phospholipase and haemolysin activities of Candida species isolated from clinical specimens. Inter J Med Biomed Res. 2012; 1:153-157.

30. Borst A, Fluit AC. High levels of hydrolytic enzymes secreted by Candida albicans isolates involved in respiratory infections. J Med Microbiol. 2003; 52:971-974.

31. Oksuz S, Sahin I, Yildirim M, Gulcan A, Yavuz T, Kaya D, Koc AN. Phospholipase and proteinase activities in different Candida species isolated from anatomically distinct sites of healthy adults. Jpn J Infect Dis. 2007; 60:280-283. 